\title{
Sunitinib mesylate inhibits proliferation of human colonic stromal fibroblasts in vitro and in vivo*
}

\author{
Zhan-huai $\mathrm{WANG}^{1,2}$, Qiong $\mathrm{LI}^{1}$, Shu-qin RUAN ${ }^{3}$, Qian XIAO ${ }^{1}$, Yue $\mathrm{LIU}^{1}$, Ye-ting HU ${ }^{1}$, \\ Li-feng $\mathrm{HU}^{1}$, Hai-yan $\mathrm{CHEN}^{1}$, Shu ZHENG ${ }^{1}$, Su-zhan ZHANG ${ }^{\dagger+1}$, Ke-feng DING ${ }^{\dagger 1,2}$ \\ $\left({ }^{1}\right.$ MOE Key Laboratory of Cancer Prevention \& Intervention, Zhejiang Provincial Key Laboratory of Molecular Biology in Medical Sciences, \\ Cancer Institute, the Second Affiliated Hospital, School of Medicine, Zhejiang University, Hangzhou 310009, China) \\ $\left({ }^{2}\right.$ Department of Oncology, the Second Affiliated Hospital, School of Medicine, Zhejiang University, Hangzhou 310009, China) \\ ( ${ }^{3}$ Department of Oncology, Chongqing Zhongshan Hospital, Chongqing 400013, China) \\ †E-mail: dingkefeng@126.com; suzhan.zhang@hotmail.com \\ Received Dec. 27, 2013; Revision accepted Apr. 16, 2014; Crosschecked June 13, 2014
}

\begin{abstract}
Objective: Cancer stromal fibroblasts are important members of the cancer microenvironment. In this study, we determined the effect of sunitinib, a small molecule tyrosine kinase inhibitor, on the primary human colonic fibroblasts. Methods: Cell cycle analysis and cell proliferation assays were performed to evaluate the inhibitory effect of sunitinib in vitro. Western-blot analysis was performed to evaluate variations in the levels of phosphorylated plateletderived growth factor receptor $\beta$ (PDGFR- $\beta$ ), Akt, and ERK proteins. Co-injection of SW620 cells and colonic fibroblasts in nude mice was employed to test anti-growth efficacy in vivo. Results: Sunitinib was found to effectively inhibit the growth of primary colonic fibroblasts. Low-dose sunitinib blocked the PDGF-BB-induced cell proliferation and PDGFR- $\beta$ signaling. Co-injection of SW620 cells and colonic fibroblasts in nude mice generated greater tumor volumes than single injection of SW620 cells. Sunitinib treatment inhibited the SW620 cell+colonic fibroblast tumor growth more effectively than treatment of 5-fluorouracil. Conclusions: Sunitinib mesylate inhibited the proliferation of primary human colonic fibroblasts through target-inhibited PDGFR signaling in vitro and in vivo.
\end{abstract}

Key words: Colon cancer, Cancer-associated fibroblasts, Sunitinib mesylate, Platelet-derived growth factor (PDGF), PDGF receptor (PDGFR)

doi: 10.1631 jzus.B1300306

Document code: A

CLC number: R735.3+4

\section{Introduction}

Colorectal cancer (CRC) is a leading cause of cancer deaths worldwide (Siegel et al., 2014). In recent years, advances in surgical management and chemotherapy have prolonged the overall survival rate of CRC patients; however, the prognosis of advanced and recurrent CRC remains poor. Thus, CRC biology should be further explored to improve the

\footnotetext{
Corresponding authors

* Project supported by the National Natural Science Foundation of China (Nos. 81071801 and 81272455) and the Zhejiang Provincial Natural Science Foundation of China (No. R2100071)

c) Zhejiang University and Springer-Verlag Berlin Heidelberg 2014
}

current treatment methods or develop new approaches. CRC cells secrete numerous growth factors and cytokines, such as the platelet-derived growth factor (PDGF) family, during tumor progression. PDGFs are disulfide-linked dimers that consist of two polypeptide chains (12.0-13.5 kDa) and designated as PDGF$A$ and PDGF-B. The five naturally occurring PDGFs (PDGF-AA, PDGF-BB, PDGF-AB, PDGF-CC, and PDGF-DD) are potent mitogens for various cancer cell types including gliomas, sarcomas, and pancreatic and prostate cancers. They possess angiogenic abilities in vivo (Heldin and Westermark, 1999; Li et al., 2000; LaRochelle et al., 2001). Two distinct signaling receptors, PDGF receptor $\alpha$ (PDGFR- $\alpha$ ) and 
PDGFR- $\beta$, used by PDGFs, have been identified (Heldin et al., 1998). PDGFR- $\alpha$ is a high-affinity receptor that interacts with all the five PDGF forms, whereas PDGFR- $\beta$ only interacts with PDGF-BB, PDGF-AB, and PDGF-DD (Bergsten et al., 2001). Recent reports have indicated that PDGFR- $\beta$ is predominantly expressed by cancer-associated stromal cells and pericytes in human colon carcinomas; PDGFR- $\beta$ expression and phosphorylation in stromal cells are related to tumor vascularity and tumor stage (Kitadai et al., 2006).

Putative cancer stroma consists of several cellular elements, among which cancer stromal fibroblast is one of the major elements. Cancer stromal fibroblasts differ from normal fibroblasts in terms of phenotype, tumor-enhancing functions, and gene expression profiles (Allinen et al., 2004; Orimo et al., 2005; Bauer et al., 2010). Moreover, tumor cells induce fibroblast activation in CRC, which consequently promotes tumor cell migration (Henriksson et al., 2011). The interaction between cancer cells and their stromal microenvironment is crucial to cancer development and progression. These specific interactions may become promising targets for new CRC therapies.

Sunitinib mesylate (SU11248, Sutent) is an orally bioavailable small molecule that inhibits multiple molecules involved in tumor growth, proliferation, and metastasis. Sunitinib targets two important receptors, namely vascular endothelial growth factor receptor (VEGFR) and PDGFR, which are expressed in various types of solid tumors (Mendel et al., 2003). Moreover, sunitinib inhibits other receptors, such as KIT, FLT3 (fms-like tyrosine kinase 3), and RET, which have key roles in solid tumors and hematologic malignancies (Chow and Eckhardt, 2007). The indications and usage of the kinase inhibitor sunitinib, approved by the US Food and Drug Administration, is for treating advanced renal cell carcinoma, gastrointestinal stromal tumor after disease progression, as well as imatinib mesylate intolerant and progressive, well-differentiated pancreatic neuroendocrine tumors in patients with locally advanced irremovable tumor or metastatic disease (Demetri et al., 2006; Motzer et al., 2007; Raymond et al., 2011).

Preclinical studies have indicated that sunitinib exhibits anti-cancer activities on colon cancer cells when combined with tumor necrosis factor-related apoptosis-inducing ligand (Ding et al., 2010). How- ever, the effect of sunitinib on colon cancer stroma remains unclear. In the present study, we determined the effect of sunitinib on primary human colonic fibroblasts from colon cancer in vitro and in vivo, as well as its underlying molecular mechanisms.

\section{Materials and methods}

\subsection{Reagents}

Sunitinib (Pfizer Inc., USA) was suspended in dimethylsulfoxide (DMSO) for in vitro studies, or in a carboxymethylcellulose (CMC) solution $(0.5 \%(5 \mathrm{~g} / \mathrm{L})$ CMC, $1.8 \%(18 \mathrm{~g} / \mathrm{L}) \mathrm{NaCl}, 0.4 \%$ Tween 80 , and $0.9 \%$ benzyl alcohol in distilled water) for in vivo experiments, to obtain $100 \mathrm{mmol} / \mathrm{L}$ stock solutions, which were then stored at $-20^{\circ} \mathrm{C}$.

5-Fluorouracil (5-FU; Sigma-Aldrich, USA) was suspended in DMSO to obtain $250 \mathrm{mmol} / \mathrm{L}$ stock concentration, which was then stored at $-20{ }^{\circ} \mathrm{C}$ and diluted in cell medium or phosphate buffered saline (PBS) for in vitro or in vivo assay, respectively.

Recombinant human PDGF-BB (PeproTech, USA) was dissolved in sterilized water that contained $0.1 \%$ bovine serum albumin to obtain $1 \mu \mathrm{g} / \mu \mathrm{l}$ stock solution, which was then stored at $-80^{\circ} \mathrm{C}$.

Antibodies used are: anti-human-Akt, antihuman-phospho-Akt (Ser473), anti-human-phosphoPDGFR- $\beta$ (Tyr751), and anti-human-GAPDH obtained from Cell Signaling Technology (Danvers, MA, USA); anti-human-vimentin, anti-mouse CD34, and anti-human-ERK2 obtained from Abcam plc (Hong Kong, China); anti-human-PDGFR- $\alpha$, antihuman-phospho-p44/42 MAPK (mitogen-activated protein kinase) (ERK1/2) (Thr202/Tyr204), antihuman-PDGFR- $\beta$, horseradish peroxidase (HRP), and conjugated anti-mouse and anti-rabbit $\operatorname{IgG}$ were obtained from Epitomics (Burlingame, USA).

\subsection{Cells and culture conditions}

The freshly dissected colon cancer tissue specimens were extensively washed, minced, and incubated in $1 \mathrm{mg} / \mathrm{ml}$ collagenase type I and $1 \mathrm{mg} / \mathrm{ml}$ hyaluronidase (Sigma-Aldrich, USA) for $2 \mathrm{~h}$ at $37^{\circ} \mathrm{C}$. The cells were separated by centrifugation and re-suspended in Iscove's modified Dulbecco's medium (Invitrogen, USA) supplemented with penicillinstreptomycin and 10\% fetal bovine serum (FBS; 
Invitrogen, USA). Early passages of the three colonic fibroblasts (F-1, F-2, and F-3) were used in the following experiments (passage 15 for all experiments).

Colon cancer cell line SW620 (Cell Bank of Type Culture Collection, Chinese Academy of Sciences, China) was maintained in Leibovitz's L-15 medium (Invitrogen, USA) supplemented with 10\% FBS, and stored at $37{ }^{\circ} \mathrm{C}$ in an incubator aerosolized with $5 \% \mathrm{CO}_{2}$.

\subsection{Cell cycle analysis}

Cell cycle was analyzed by flow cytometry. Subconfluent cultures $(24 \mathrm{~h})$ of sunitinib-treated colonic fibroblasts ( 1 or $8 \mu \mathrm{mol} / \mathrm{L}$ ) were trypsinized, washed with PBS, fixed in $70 \%$ ethanol, and stained with propidium iodide $(50 \mu \mathrm{g} / \mathrm{ml})$ in the presence of $\mathrm{MgCl}_{2}(5 \mathrm{mmol} / \mathrm{L})$ and RNase A $(10 \mu \mathrm{g} / \mathrm{ml})$ in Tris- $\mathrm{HCl}(\mathrm{pH} 7.5 ; 10 \mathrm{mmol} / \mathrm{L})$. DNA content was determined using a FACSCalibur flow cytometer (Becton, Dickinson and Company, USA) with Modfit software.

\subsection{Cell proliferation assay}

Cell proliferation assays were performed by using CellTiter 96 Aqueous One Solution Cell Proliferation Assay that contained MTS (Promega Corporation, USA). Each well of the 96-well plates was seeded with 2000-3000 colonic fibroblasts or 3000 SW620 cells in a culture medium that contained $10 \%$ FBS. The cells were treated with different concentrations of 5-FU or sunitinib after overnight culture. MTS was added to the cells in accordance with the protocol of the supplier $72 \mathrm{~h}$ after the treatments. Absorbance was then measured at $490 \mathrm{~nm}$ using an automated enzyme-linked immunosorbent assay (ELISA) plate reader.

The medium in the overnight cultured cells in the 96-well plates was replaced by a serum-free medium that contained PDGF-BB $(10 \mathrm{ng} / \mathrm{ml})$ and various sunitinib concentrations to evaluate the effects of growth factors PDGF-BB and tyrosine kinase inhibitor sunitinib on the colonic fibroblast growth. MTS assay was conducted $72 \mathrm{~h}$ after treatment.

\subsection{Western-blot analysis}

Subconfluent colonic fibroblasts were cultured overnight in a serum-free medium, pretreated with various sunitinib concentrations for $2 \mathrm{~h}$, and stimu- lated with PDGF-BB (10 ng/ml) for $20 \mathrm{~min}$. The cells were washed with ice-cold PBS and lysed in a protein lysis buffer (Pierce Chemical Company, USA) supplemented with protease/phosphatase inhibitor cocktails (Pierce Chemical Company) in accordance with the protocol of the manufacturer. Protein concentrations were determined using the BCA protein assay kit (Pierce Chemical Company). The samples were separated on $8 \%-12 \%$ sodium dodecyl sulfatepolyacrylamide gels, transferred to polyvinylidene difluoride (PVDF) membranes (EMD Millipore, USA), blocked, and detected by appropriate primary and secondary antibodies before visualization using a chemiluminescence detection kit for HRP.

\subsection{Animals and orthotopic tumor implantation}

This study was approved by the Institutional Animal Ethical Committee of the Second Affiliated Hospital of Zhejiang University, China. Animal care was performed in accordance with the institutional guidelines.

Six-week-old male BALB/c athymic nude mice (SLAC Laboratory Animal Co. Ltd., Shanghai, China) were used for tumorigenesis assay. SW620 cells, (SW620+F-1) cells, or $(\mathrm{SW} 620+\mathrm{F}-2)$ cells $\left(1 \times 10^{6}\right.$ cells of each cell line) mixed in Matrigel matrix (BD Biosciences, USA) were injected subcutaneously into nude mice at random. Each group of mice was subdivided into control and treatment subgroups $(n=6)$. Four days after injection, the mice were treated with a vehicle of sunitinib $(40 \mathrm{mg} / \mathrm{kg}$; oral route) or $5-\mathrm{FU}$ $(20 \mathrm{mg} / \mathrm{kg}$; intraperitoneal injection) in a 5 -d-on/ 2-d-off treatment cycle (5/2 schedule) based on the subgroup. The length $(l)$ and width $(w)$ of the tumor and body weight of the mice were measured with calipers twice a week. The tumor volume $(V)$ was calculated as $V=l w^{2} / 2$.

\subsection{Immunohistochemical analysis}

Tumors from both control and treatment groups were excised after $5 \mathrm{~d}$, fixed with formalin, embedded in paraffin, and cut into serial 4- $\mu \mathrm{m}$ sections. Tissue sections were stained with antibodies to detect primary human colonic fibroblasts using anti-human vimentin, whereas tumor microvascular density (MVD) was determined using anti-mouse CD34. The sections were visualized using the EnVision Detection System (Dako, Glostrup, Denmark). Three tissue 
sections from each of the six mice in each group were evaluated. Tissue sections were examined under a light microscope to identify five regions with the highest MVD $(100 \times)$, and then recorded to quantify tumor MVD (200×). Tissue sections were also examined $(100 \times)$ to identify five regions with the highest vimentin-positive cells, and then recorded to quantify primary human colonic fibroblasts $(400 \times)$. The results were then statistically analyzed.

\subsection{Statistical analysis}

The results were expressed as mean \pm standard error (SE). The differences between each group, such as cellular growth rate, were analyzed by paired Student's $t$-test and based on the mouse body weight. Quantifications of the Western-blot results, tumor volumes, $\mathrm{CD} 34^{+}$areas, and vimentin-positive cells were analyzed using one-way analysis of variance (ANOVA) or non-parametric test. $P<0.05$ was considered as statistically significant.

\section{Results}

\subsection{Effects of sunitinib and 5-FU on the growth of SW620 cells and human colonic fibroblasts}

Cell proliferation assays were carried out in the presence of varying sunitinib concentrations for $72 \mathrm{~h}$ to determine the inhibitory effect of sunitinib on colonic fibroblasts and SW620 colon cancer cells in vitro. Significant growth inhibitory effects on SW620 cells and colonic fibroblasts were observed in a dose-dependent manner (Fig. 1a). Moreover, the effects of 5-FU on the growth of colonic fibroblasts and SW620 cells for $72 \mathrm{~h}$ were determined via MTS assay. 5-FU significantly inhibited SW620 cell growth in a dose-dependent manner, but had no effect on colonic fibroblasts even at $250 \mu \mathrm{mol} / \mathrm{L}$ (Fig. 1b).

\subsection{Cell cycle analysis}

Cell cycle analysis $(24 \mathrm{~h})$ of 1 or $8 \mu \mathrm{mol} / \mathrm{L}$ sunitinib-treated colonic fibroblasts was performed via flow cytometry to evaluate the inhibitory effect of sunitinib against colonic fibroblast proliferation. The result indicates a dose-dependent decrease in $\mathrm{S}$ and $\mathrm{G} 2 / \mathrm{M}$ phases as well as an accumulation of cells in the $\mathrm{G} 0 / \mathrm{G} 1$ phase. No significant cell apoptosis was detected even at $8 \mu \mathrm{mol} / \mathrm{L}$ sunitinib (Figs. 1c-1e).

\subsection{Expression levels of PDGFR- $\alpha$ and PDGFR- $\beta$ in SW620 cells and human colonic fibroblasts}

PDGFR- $\alpha$ and PDGFR- $\beta$ expression levels in colonic fibroblasts and SW620 cells were determined by Western-blot analysis. Anti-PDGFR- $\alpha(110 \mathrm{kDa})$ and PDGFR- $\beta$ (190 kDa) immunoreactive proteins were found in all colonic fibroblast protein extracts. In contrast to PDGFR- $\alpha$ expression, PDGFR- $\beta$ was highly expressed in all colonic fibroblasts. Neither PDGFR- $\alpha$ nor PDGFR- $\beta$ was expressed in SW620 cells (Fig. 2a).

\subsection{Blocking of PDGF-BB-induced human colonic fibroblast proliferation by sunitinib}

Given that sunitinib mesylate is a specific inhibitor of the PDGFR, we subsequently assessed the effect of PDGF on colonic fibroblast proliferation, with or without sunitinib. Incubating with $10 \mathrm{ng} / \mathrm{ml}$ PDGF-BB significantly stimulated colonic fibroblast growth in the serum-free culture medium for $72 \mathrm{~h}$, whereas co-incubating with $10 \mathrm{ng} / \mathrm{ml}$ PDGF-BB and various sunitinib concentrations, even at $50 \mathrm{nmol} / \mathrm{L}$, effectively blocked PDGF-induced growth of colonic fibroblasts (Figs. 2b-2d).

\subsection{Inhibition of PDGF-BB-induced PDGFR- $\beta$} signaling in human colonic fibroblasts by sunitinib

The total levels of phosphorylated PDGFR- $\beta$, Akt, and ERK proteins in colonic fibroblasts treated with PDGF and/or sunitinib were examined to investigate the mechanisms by which sunitinib inhibits PDGF-induced colonic fibroblast proliferation. The total protein levels of PDGFR- $\beta$, Akt, and ERK $1 / 2$ in all three colonic fibroblasts were not significantly changed; however, stimulation by $10 \mathrm{ng} / \mathrm{ml}$ PDGFBB alone significantly increased the phosphorylated levels of the aforementioned proteins. Meanwhile, pretreatment with sunitinib at various concentrations $(0.05,0.10,0.20,0.50$, and $1.00 \mu \mathrm{mol} / \mathrm{L})$ suppressed the PDGF-stimulated phosphorylation in a dosedependent manner (Fig. 3). Fold changes in phosphorylated protein (RR:) between the treatment and the control groups were determined by three independent experiments and normalized by the total protein level. 
(a)
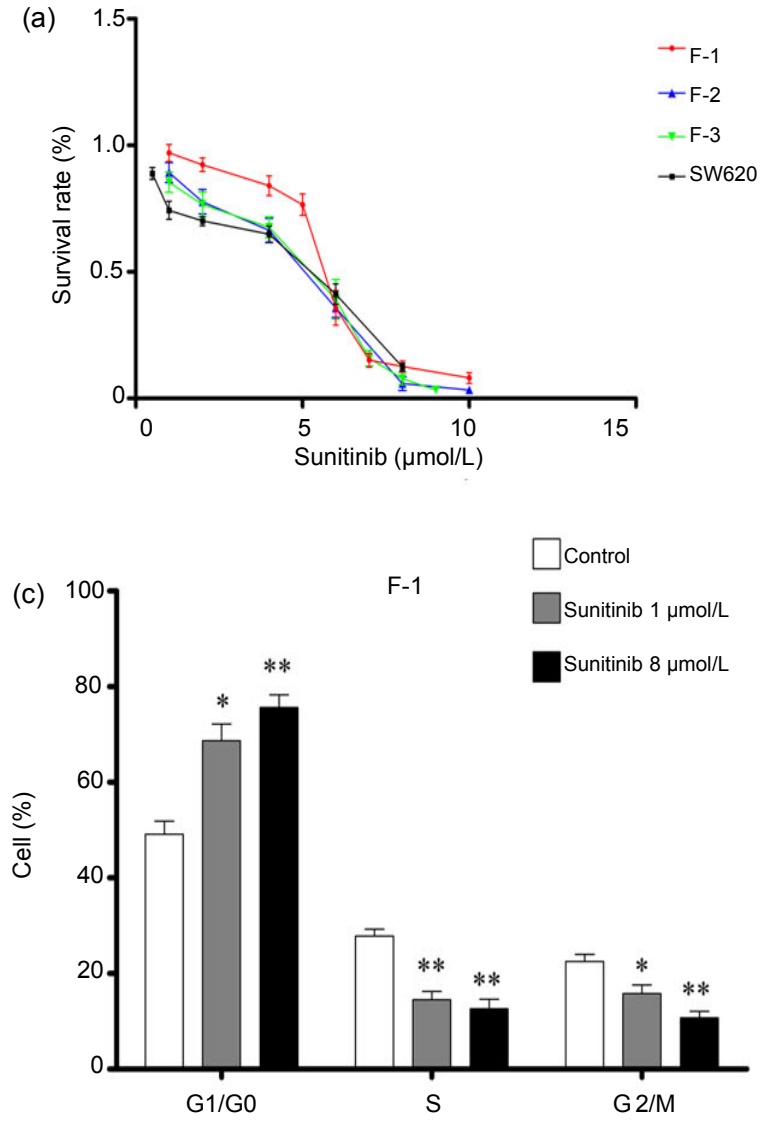

(b)
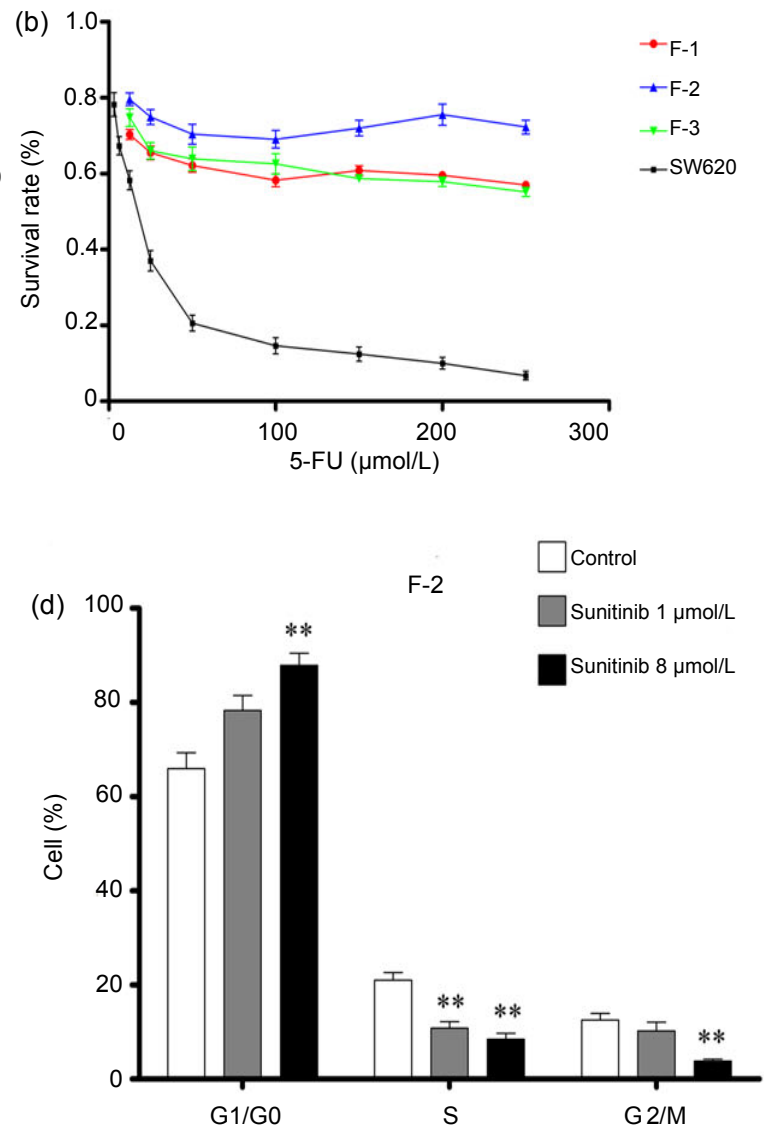

F-3

$\square$ Control

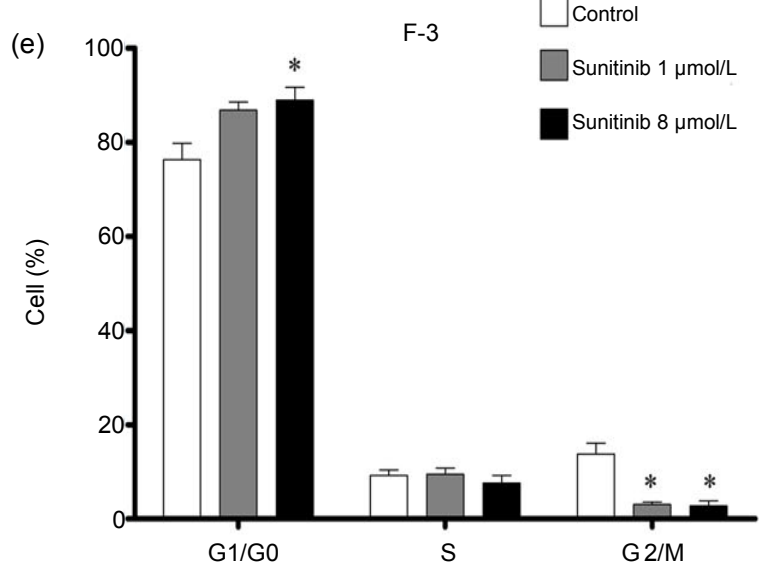

Fig. 1 Cell proliferation assay carried out in the presence of sunitinib (a) or 5-FU (b) for $72 \mathrm{~h}$ and cell circle analyses of colonic fibroblasts F-1 (c), F-2 (d), and F-3 (e) treated with sunitinib for 24 h

Sunitinib inhibited the proliferation of SW620 cells and colonic fibroblasts (F-1, F-2, and F-3) in a dose-dependent manner (a), while 5-FU effectively inhibited SW620 cell growth, but exhibited no significant effect on colonic fibroblasts (F-1, F-2, and F-3) (b). As shown in (c)-(e), results of cell cycle analysis indicate a decrease in S and G2/M phases, as well as cell accumulation in $\mathrm{G} 0 / \mathrm{G} 1$ phase by the sunitinib treatment. Data are presented as mean $\pm \mathrm{SE}(n=3)$. ${ }^{*} P<0.05,{ }^{* *} P<0.01$, vs. control 
(a)
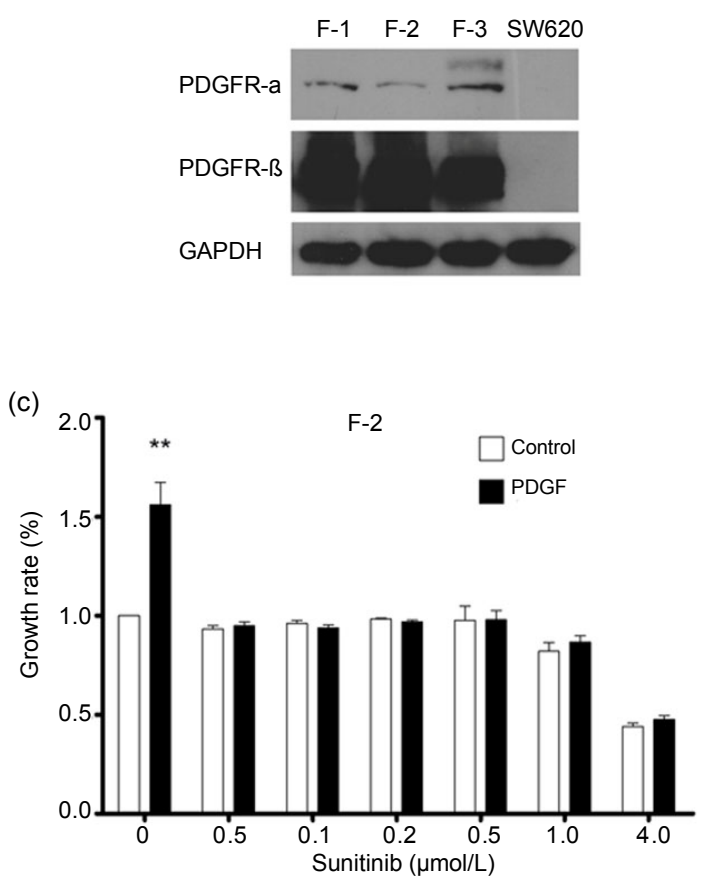
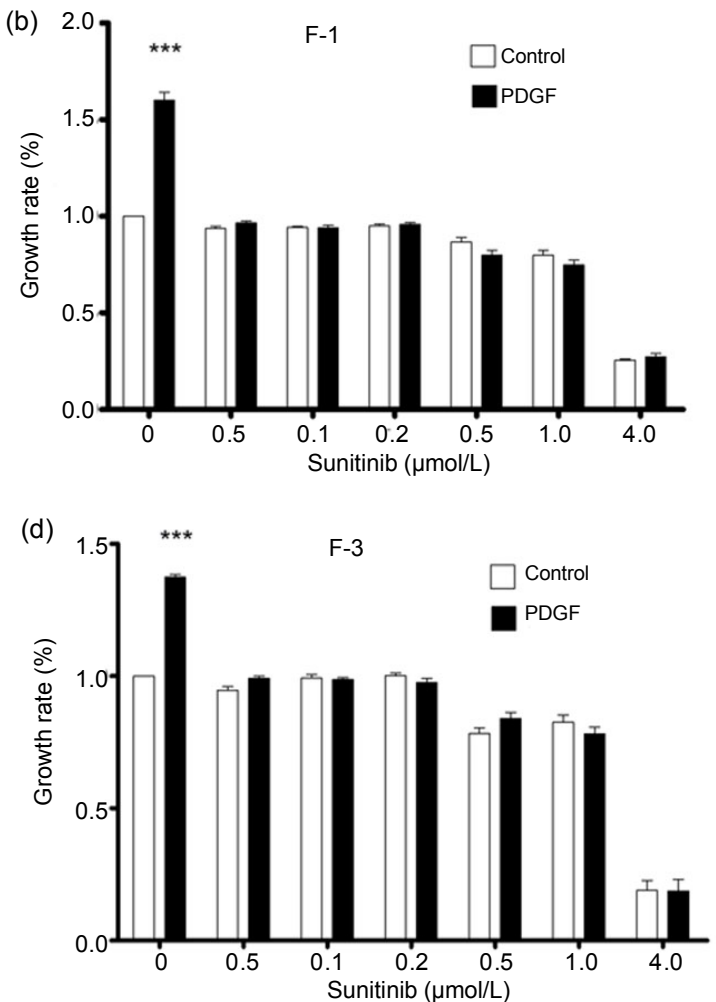

Fig. 2 Expression levels of PDGFR- $\alpha$ and PDGFR- $\beta$ in SW620 cells and human colonic fibroblasts (a) and blocking effects of sunitinib on the proliferation of PDGF-BB-induced human colonic fibroblasts F-1 (b), F-2 (c), and F-3 (d) (a) PDGFR- $\alpha$ and PDGFR- $\beta$ expression levels in SW620 cells and colonic fibroblasts (F-1, F-2, and F-3) were determined by Western-blot analysis. (b-d) Cell proliferation assay suggested that $10 \mathrm{ng} / \mathrm{ml}$ PDGF-BB significantly stimulated the growth of all colonic fibroblasts in the serum-free culture medium for $72 \mathrm{~h}$. Sunitinib blocked PDGF-BB-induced colonic fibroblast growth even at $50 \mathrm{nmol} / \mathrm{L}$ concentration. Data are presented as mean $\pm \mathrm{SE}(n=3) .{ }^{*} P<0.05,{ }^{* *} P<0.01,{ }^{* * *} P<0.001$, vs. control

\subsection{Effect of sunitinib on tumor growth in mice xenograft models}

We developed a mouse xenograft model that bore subcutaneous SW620 cells and colonic fibroblasts to determine the contribution of colonic fibroblasts to tumor growth and the effect of sunitinib in vivo. SW620 cells or SW620 cells mixed with F-1 cells (SW620+F-1) or F-2 cells (SW620+F-2) in a 1:1 ratio were inoculated subcutaneously into immunocompromised nude mice to develop the mouse xenograft model. Each group was divided into three subgroups administered with a vehicle, sunitinib, or 5-FU. F-3 cells were not included in the in vivo tests because the life span is too short to obtain sufficient numbers of cells.

SW620 cells mixed with either F-1 or F-2 cells inoculated into nude mice generated significantly greater tumor volumes than inoculation with SW620 cells alone $(P<0.05)$, whereas nude mice inoculated with colonic fibroblasts alone did not generate any tumor (data not shown). These results indicate that F-1 and F-2 cells significantly enhanced SW620 tumor cell growth. By contrast, sunitinib significantly inhibited tumor growth in all three groups, namely, SW620, SW620+F-1, and SW620+F-2 $(P<0.05)$, whereas 5-FU administration resulted in moderate tumor growth inhibition in the SW620+F-1 and SW620+F-2 groups $(P<0.05$; Fig. 4).

The side-effects of sunitinib and 5-FU were also evaluated based on the in vivo assay. No mouse died in any of the groups. In each group, the average body weights of the mice in the sunitinib or 5-FU subgroups were not significantly different from those of the mice in the control subgroups (Table 1). 
(a)

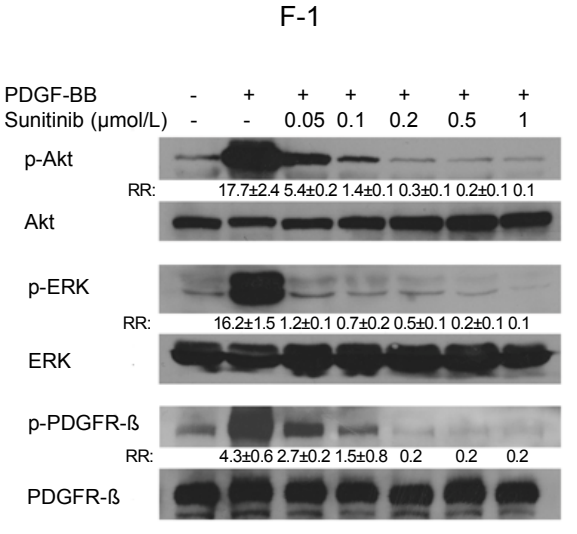

(c)

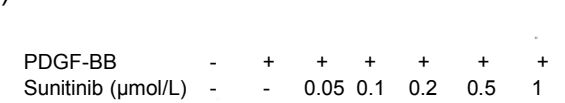

Sunitinib $(\mu \mathrm{mol} / \mathrm{L}) \quad-\quad \begin{array}{llllll}0 & 0.05 & 0.1 & 0.2 & 0.5 & 1\end{array}$

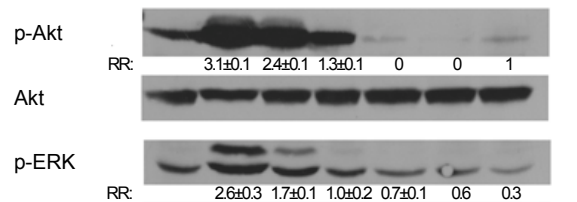

ERK

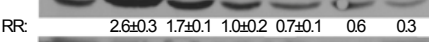

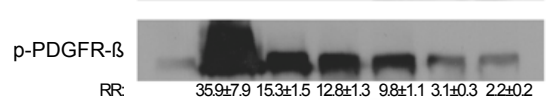

PDGFR-ß

(b)

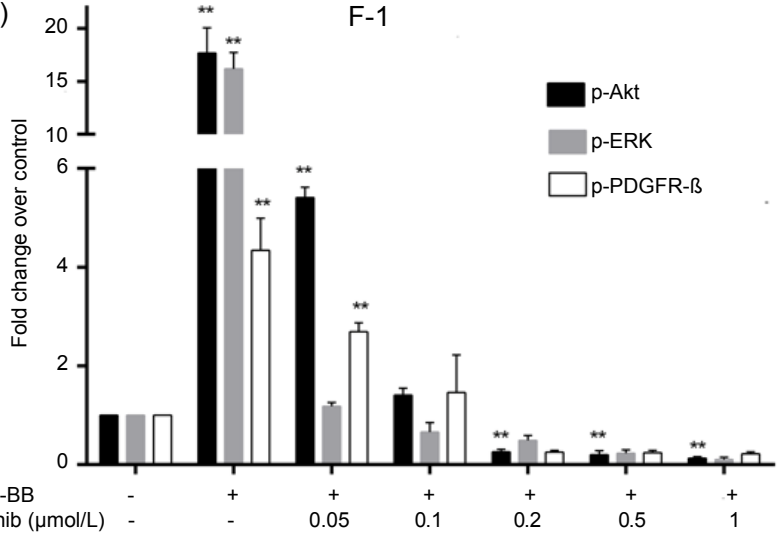

(1)

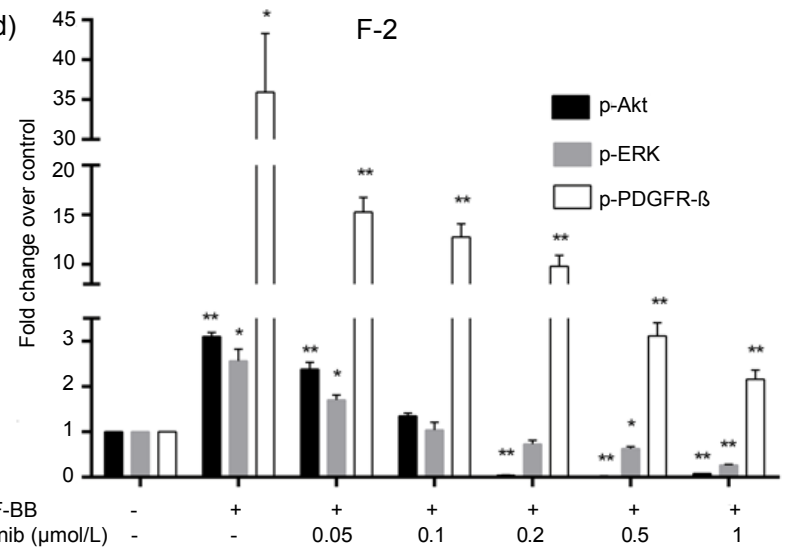

(e)

F-3
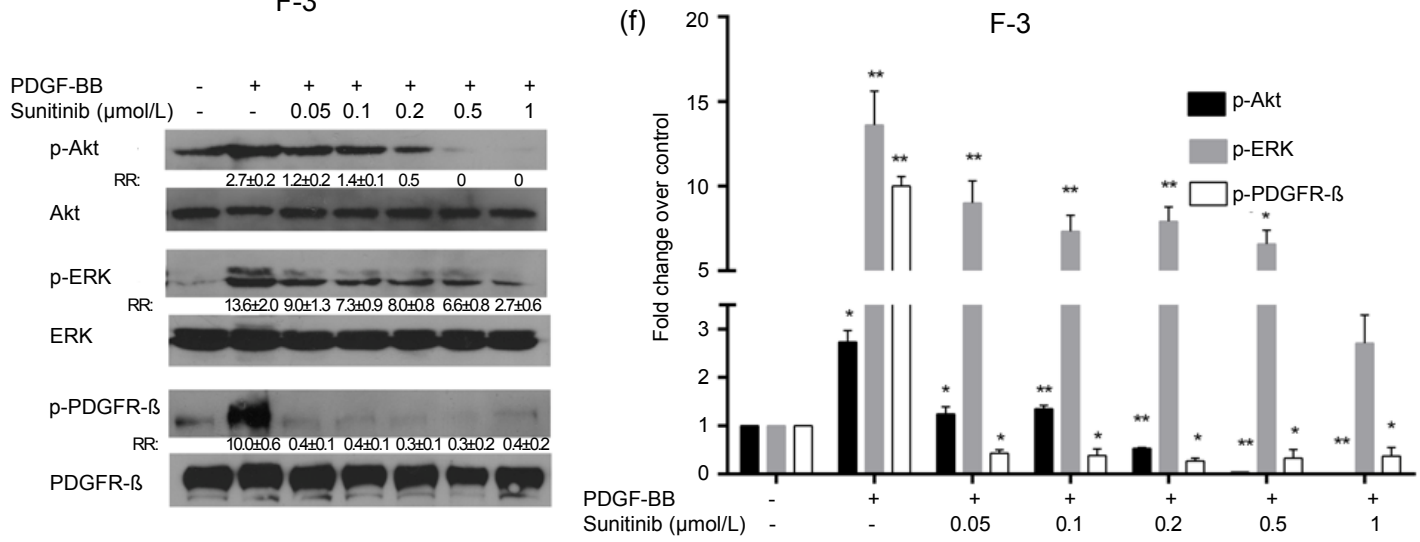

Fig. 3 Inhibition of PDGF-BB-induced PDGFR- $\beta$ signaling in human colonic fibroblasts by sunitinib The stimulation induced by $10 \mathrm{ng} / \mathrm{ml}$ PDGF-BB increased the amounts of phosphorylated PDGFR- $\beta$, Akt, and ERK proteins in colonic fibroblasts F-1 (a, b), F-2 (c, d), and F-3 (e, f). Pretreatment with various sunitinib concentrations suppressed the phosphorylated levels of the aforementioned proteins in a dose-dependent manner. Fold changes in phosphorylated protein (RR:) between the treatment and the control groups were determined by three independent experiments and normalized by the total protein level. Data are presented as mean \pm SE. ${ }^{*} P<0.05,{ }^{* *} P<0.01$, vs. control 
(a)

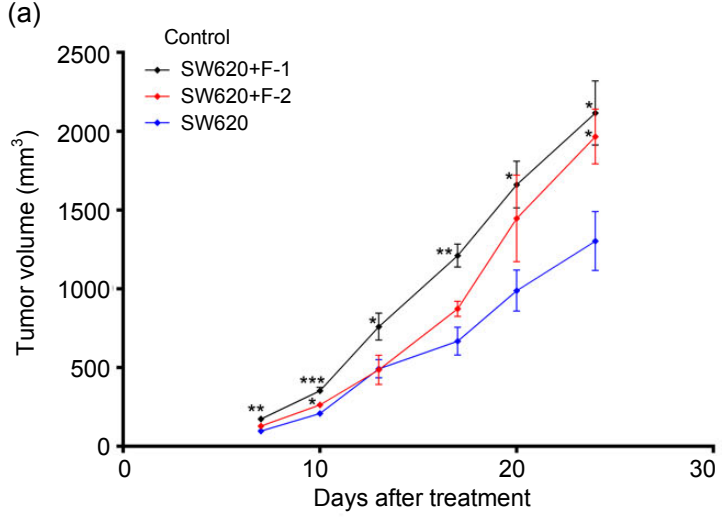

(c)

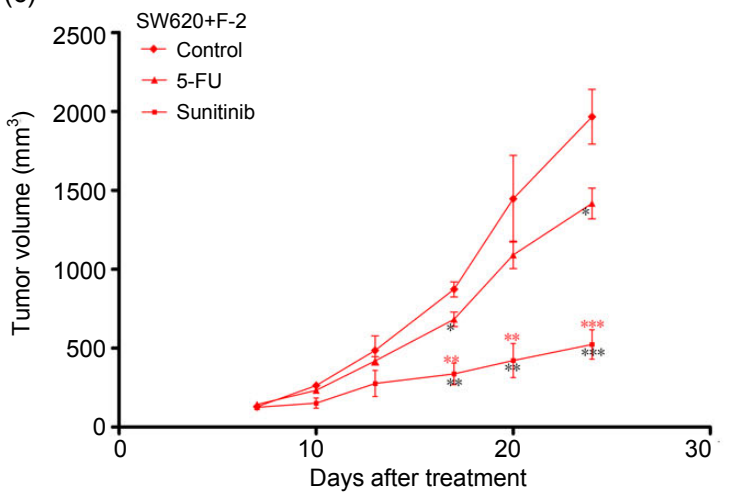

(b)

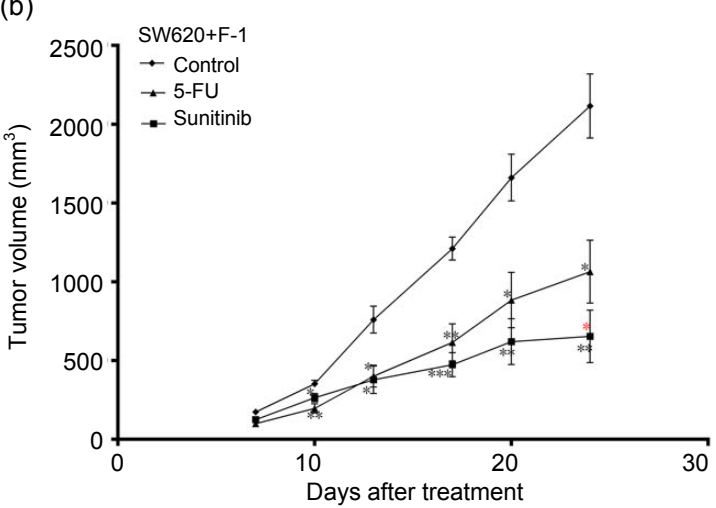

(d)

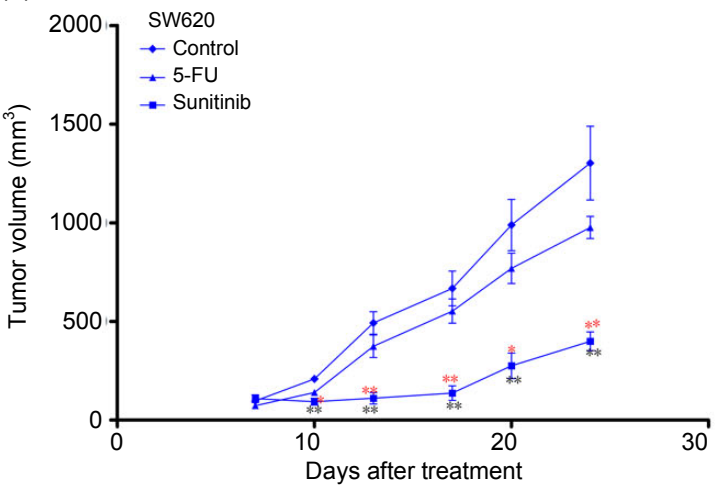

Fig. 4 Tumor volumes of the mice injected with SW620, SW620+F-1, or SW620+F-2 cells under different treatments

Each group was divided into the following subgroups: control, sunitinib, and 5-FU. (a) SW620 cells mixed with either F-1 or F-2 cells generated greater tumor volumes compared with those from SW620 cells alone. (b, c) In the SW620+F-1 and SW620+F-2 groups, both sunitinib and 5-FU significantly inhibited tumor growth; however, sunitinib more effectively inhibited tumor growth than 5-FU. (d) In the SW620 cell group, sunitinib significantly inhibited tumor growth compared with 5-FU and the control. Data are presented as mean \pm SE $(n=6) .{ }^{*} P<0.05$, ${ }^{* *} P<0.01,{ }^{* * *} P<0.001$; the black ${ }^{*}$ indicates comparison of the treatment subgroup with the control subgroup; the red $^{*}$ denotes comparison of sunitinib with 5-FU

Table 1 Antitumor effects of sunitinib and 5-FU

\begin{tabular}{clccc}
\hline Group & Treatment & Body weight $(\mathrm{g})$ & Tumor incidence $(\%)$ & Tumor volume $\left(\mathrm{mm}^{3}\right)$ \\
\hline SW620 & Control & $23.92 \pm 0.87$ & 100 & $1303 \pm 186.9$ \\
& 5-FU & $22.80 \pm 0.65$ & 100 & $826.4 \pm 70.9$ \\
& Sunitinib & $25.28 \pm 1.13$ & 100 & $523 \pm 92.9^{* *}$ \\
SW620+F-1 & Control & $22.04 \pm 0.51$ & 100 & $2116 \pm 203.4$ \\
& 5-FU & $20.88 \pm 0.82$ & 100 & $1064 \pm 199.9^{*}$ \\
& Sunitinib & $24.40 \pm 0.76$ & 100 & $653.6 \pm 166.5^{* *}$ \\
SW620+F-2 & Control & $21.64 \pm 0.53$ & 100 & $1966 \pm 173.8$ \\
& 5-FU & $20.50 \pm 1.62$ & 100 & $1417 \pm 97.73^{*}$ \\
& Sunitinib & $24.24 \pm 1.19$ & 100 & $399.2 \pm 47.7^{* * *}$ \\
\hline
\end{tabular}

Data are presented as mean $\pm \operatorname{SE}(n=6) .{ }^{*} P<0.05,{ }^{* *} P<0.01,{ }^{* * *} P<0.001$, vs. control 


\subsection{Histopathologic analysis of mouse xenograft tumor}

The total numbers of colonic fibroblasts were determined by staining SW620+colonic fibroblast tumors with anti-human vimentin. Sunitinib treatment significantly decreased the number of colonic fibroblasts compared with the control $(P<0.001)$ and 5-FU treatment $(P<0.001)$, whereas 5-FU treatment did not significantly change the numbers of colonic fibroblasts (Fig. 5).
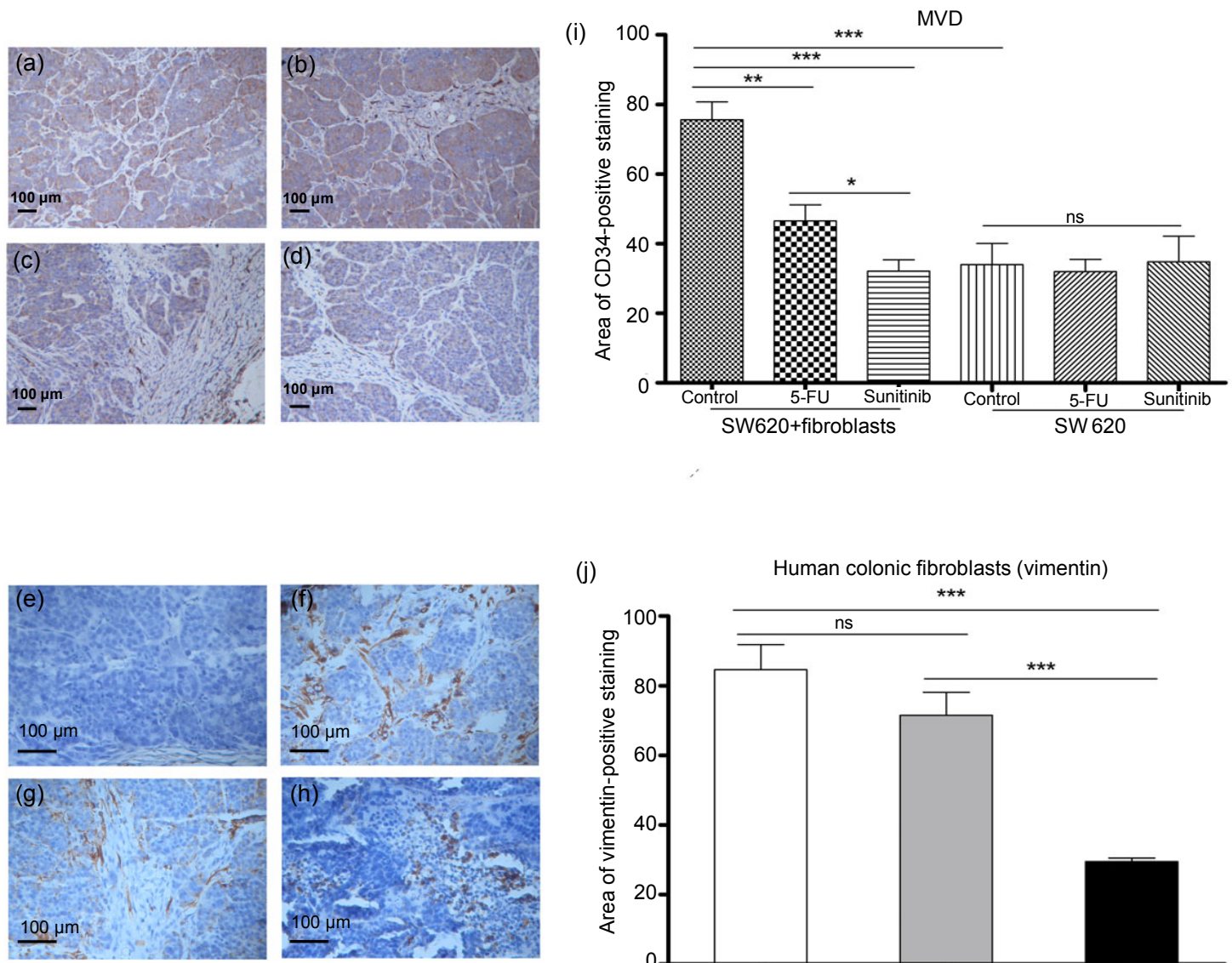

Tumor MVD was evaluated by CD34 staining. Mixing colonic fibroblasts with SW620 cells significantly increased the tumor MVD compared with using SW620 cells only $(P<0.001)$. In the SW620+ colonic fibroblast group, sunitinib significantly decreased the tumor MVD compared with the control $(P<0.001)$ and 5-FU $(P<0.05)$. However, neither sunitinib nor 5-FU treatment significantly decreased tumor MVD in the SW620 cell group compared with that in the control group (Fig. 5). (j)

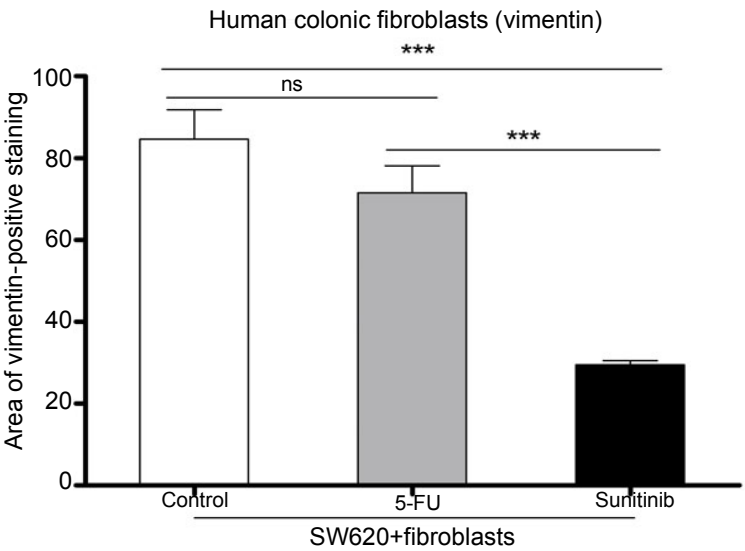

Fig. 5 Results of histopathologic analysis of mouse xenograft tumor

(a, e) SW620 cell control; (b, f) SW620 cell+colonic fibroblast control; (c, g) SW620 cells+colonic fibroblasts treated with 5-FU; (d, h) SW620 cells+colonic fibroblasts treated with sunitinib. (i) MVD was quantified according to the CD34-positive areas (a-d) at 200× magnification. Co-mixing colonic fibroblasts with SW620 cells significantly increased tumor MVD compared with using SW620 cells alone $(P<0.001)$. In the SW620 cell+colonic fibroblast group, sunitinib treatment significantly decreased tumor MVD compared with the control $(P<0.001)$ and 5-FU treatment $(P<0.05)$. However, neither sunitinib nor 5-FU treatment significantly decreased tumor MVD in the SW620 cell group compared with the control group. (j) Primary fibroblasts were quantified according to vimentin-positive $(\mathrm{e}-\mathrm{h})$ areas at $400 \times$ magnification. Sunitinib treatment significantly decreased the numbers of colonic fibroblasts compared with the control $(P<0.001)$ and 5-FU treatment $(P<0.001)$, whereas 5-FU treatment exhibited no significant effect on the numbers of colonic fibroblasts. Data are presented as mean \pm SE $(n=6) .{ }^{*} P<0.05 ;{ }^{* *} P<0.01 ;{ }^{* * *} P<0.001$ 


\section{Discussion}

Cancer progression is increasingly recognized as being dependent not only on the cancer cells themselves, but also on stromal fibroblasts in cancer microenvironments (Mueller et al., 2007b; Cui et al., 2010; Henriksson et al., 2011; Tyan et al., 2011; Zhang et al., 2011). Stromal fibroblasts modulate tumorigenic activities of cancer cells, such as proliferation, apoptosis, and angiogenesis (Tomasek et al., 2002; Kalluri, 2003; Micke and Ostman, 2005). Therefore, it is necessary to determine the specific interaction between cancer cells and stromal fibroblasts. Continuous cell line growth may exhibit differences in cell proliferation and protein expression; thus, performing experiments during early passage is important. In the present study, we used three low-passage primary human colonic fibroblasts (F-1, F-2, and F-3) cultured from an excised tissue specimen of colon cancer. Similar experiments were performed on these low-passage colonic fibroblasts to determine their common features. However, primary cells have a short life-span, thereby limiting the experimental repeatability. In the present study, the three primary colonic fibroblasts had short life-spans. Although the life-span of F-3 cells was too short to obtain sufficient numbers of cells to conduct animal tests, the biological properties of the three colonic fibroblasts were extremely similar. Thus, the animal experiments on F-1 and F-2 cells may represent their common features.

Several studies have been carried out to target stromal fibroblasts using tyrosine kinase inhibitor, such as dasatinib or imatinib (Mueller et al., 2007a; Gioni et al., 2008; Haubeiss et al., 2010). Mueller et al. (2007a) showed that imatinib inhibits proliferation and modulates cytokine expression of human cancerassociated fibroblasts from colorectal metastases. However, the microenvironments of primary and metastatic lesions of colon cancer are dissimilar. Our results showed that the tyrosine kinase inhibitor sunitinib exhibits an inhibitory effect against human colonic fibroblasts from primary colon cancer both in vitro and in vivo.

We initially investigated the effect of sunitinib on human colonic fibroblast growth by using $5-\mathrm{FU}$ as control. Although 5-FU is a widely used agent in the clinical management of colon cancer, the inhibitory effects of sunitinib and 5-FU on colonic fibroblasts are significantly different. Cell proliferation assay indicated that sunitinib effectively inhibited human colonic fibroblast proliferation, whereas 5-FU showed no significant effect (Fig. 1). Given that fibroblasts are mainly stromal cells that are different from epithelial cells, we considered that the drug sensitivity of cancer cells and cancer stromal cells may be completely different. Furthermore, microscopic observations showed that the inhibitory effect of sunitinib on colonic fibroblasts was cytostatic (not cytotoxic), which was confirmed by the cell cycle analysis by flow cytometry (Fig. 1).

Subsequently, we confirmed that PDGFR- $\alpha$ and PDGFR- $\beta$, which bind PDGF-BB, were expressed in the three primary fibroblasts. In addition, PDGF-BB stimulated colonic fibroblast proliferation in serumfree medium. Moreover, $50 \mathrm{nmol} / \mathrm{L}$ sunitinib inhibited PDGF-BB-mediated colonic fibroblast proliferation (Fig. 2). The dose-dependent inhibitory effects of sunitinib on the PDGF-BB-mediated phosphorylation of PDGFR- $\beta$, ERK1/2, and Akt pathways were demonstrated to elucidate the mechanisms of the inhibitory action of sunitinib (Fig. 3). PDGFR signaling pathways are important in stromagenesis and cancer progression, and thus, PDGFR signaling inhibition by sunitinib may be significant in tumor stromal targeted treatment. Given that sunitinib is a multitarget tyrosine kinase inhibitor, we investigated the effect of sunitinib on VEGFR, which is one of its important targets in primary fibroblasts. However, the Western-blot analysis indicated that none of the VEGFR signaling receptors, such as VEGFR1, VEGFR2, and VEGFR3, were expressed in these fibroblasts (data not shown).

Recent studies have shown that co-injecting stromal fibroblasts and tumor cells is more consistent in promoting cell growth than injecting tumor cells alone (Orimo et al., 2005; Liao et al., 2009). The co-injection model provides the basis for studying the interactions between stromal fibroblasts and cancer cells in vivo. Thus, in vivo assay was performed using the SW620 cell+colonic fibroblast co-injection model to evaluate the effect of sunitinib on colonic fibroblasts. The results showed that primary human colonic fibroblasts enhanced the proliferation of SW620 cells. The nude mice that bore colonic fibroblasts + SW620 tumor cells generated larger tumor volumes 
than the mice with only SW620 tumor cells (Fig. 4). Histopathologic analysis confirmed that the MVD of colonic fibroblasts+SW620 tumor cells was significantly increased compared with that of SW620 tumor cells alone (Fig. 5). Treatment with sunitinib inhibited colonic fibroblasts + SW620 tumor cell growth more effectively than the 5-FU treatment. Moreover, sunitinib influenced the structure of colonic SW620+ fibroblasts tumor cells. Histopathologic analysis that used vimentin-positive staining indicates that tumor MVD and the numbers of colonic fibroblasts were significantly decreased after the sunitinib treatment (Fig. 5).

In conclusion, we demonstrated that small molecular tyrosine kinase inhibitor sunitinib mesylate inhibited proliferation of primary human colonic fibroblasts from colon cancer in vitro and in vivo. Sunitinib-targeted treatment also inhibited PDGFR signaling of colonic fibroblasts by PDGF-BB stimulation. Our results indicate that sunitinib may be valuable in treating colon cancer by suppressing stromal fibroblasts. The effect of sunitinib on stromal response can augment current chemotherapy of colon cancer by establishing a synergistic approach.

\section{Acknowledgements}

We are grateful to Dr. Kan-lun XU, Dr. Xian ZHONG, Prof. Xun HU, and Prof. Rong-zhen XU for their assistance and suggestions.

\section{Compliance with ethics guidelines}

Zhan-huai WANG, Qiong LI, Shu-qin RUAN, Qian XIAO, Yue LIU, Ye-ting HU, Li-feng HU, Hai-yan CHEN, Shu ZHENG, Su-zhan ZHANG, and Ke-feng DING declare that they have no conflict of interest.

All institutional and national guidelines for the care and use of laboratory animals were followed.

\section{References}

Allinen, M., Beroukhim, R., Cai, L., et al., 2004. Molecular characterization of the tumor microenvironment in breast cancer. Cancer Cell, 6(1):17-32. [doi:10.1016/j.ccr. 2004.06.010]

Bauer, M., Su, G., Casper, C., et al., 2010. Heterogeneity of gene expression in stromal fibroblasts of human breast carcinomas and normal breast. Oncogene, 29(12): 1732-1740. [doi:10.1038/onc.2009.463]

Bergsten, E., Uutela, M., Li, X., et al., 2001. PDGF-D is a specific, protease-activated ligand for the PDGF ß-receptor. Nat. Cell Biol., 3(5):512-516. [doi:10.1038/ 35074588]
Chow, L.Q., Eckhardt, S.G., 2007. Sunitinib: from rational design to clinical efficacy. J. Clin. Oncol., 25(7):884-896. [doi:10.1200/JCO.2006.06.3602]

Cui, L., Ohuchida, K., Mizumoto, K., et al., 2010. Prospectively isolated cancer-associated $\mathrm{CD} 10^{+}$fibroblasts have stronger interactions with $\mathrm{CD} 133^{+}$colon cancer cells than with CD133- cancer cells. PLoS ONE, 5(8):e12121. [doi:10.1371/journal.pone.0012121]

Demetri, G.D., van Oosterom, A.T., Garrett, C.R., et al., 2006. Efficacy and safety of sunitinib in patients with advanced gastrointestinal stromal tumour after failure of imatinib: a randomised controlled trial. Lancet, 368(9544):13291338. [doi:10.1016/S0140-6736(06)69446-4]

Ding, W., Cai, T., Zhu, H., et al., 2010. Synergistic antitumor effect of trail in combination with sunitinib in vitro and in vivo. Cancer Lett., 293(2):158-166. [doi:10.1016/j.canlet. 2010.01.005]

Gioni, V., Karampinas, T., Voutsinas, G., et al., 2008. Imatinib mesylate inhibits proliferation and exerts an antifibrotic effect in human breast stroma fibroblasts. Mol. Cancer Res., 6(5):706-714. [doi:10.1158/1541-7786.MCR-070355]

Haubeiss, S., Schmid, J.O., Murdter, T.E., et al., 2010. Dasatinib reverses cancer-associated fibroblasts (CAFs) from primary lung carcinomas to a phenotype comparable to that of normal fibroblasts. Mol. Cancer, 9(1):168. [doi:10.1186/1476-4598-9-168]

Heldin, C.H., Westermark, B., 1999. Mechanism of action and in vivo role of platelet-derived growth factor. Physiol. Rev., 79(4):1283-1316.

Heldin, C.H., Ostman, A., Ronnstrand, L., 1998. Signal transduction via platelet-derived growth factor receptors. Biochim. Biophys. Acta-Rev. Cancer, 1378(1):F79-F113. [doi:10.1016/S0304-419X(98)00015-8]

Henriksson, M.L., Edin, S., Dahlin, A.M., et al., 2011. Colorectal cancer cells activate adjacent fibroblasts resulting in FGF1/FGFR3 signaling and increased invasion. Am. J. Pathol., 178(3):1387-1394. [doi:10. 1016/j.ajpath.2010.12.008]

Kalluri, R., 2003. Basement membranes: structure, assembly and role in tumour angiogenesis. Nat. Rev. Cancer, 3(6): 422-433. [doi:10.1038/nrc1094]

Kitadai, Y., Sasaki, T., Kuwai, T., et al., 2006. Expression of activated platelet-derived growth factor receptor in stromal cells of human colon carcinomas is associated with metastatic potential. Int. J. Cancer, 119(11):25672574. [doi:10.1002/ijc.22229]

LaRochelle, W.J., Jeffers, M., McDonald, W.F., et al., 2001. PDGF-D, a new protease-activated growth factor. Nat. Cell Biol., 3(5):517-521. [doi:10.1038/35074593]

Li, X., Ponten, A., Aase, K., et al., 2000. PDGF-c is a new protease-activated ligand for the PDGF alpha-receptor. Nat. Cell Biol., 2(5):302-309. [doi:10.1038/35010579]

Liao, D., Luo, Y., Markowitz, D., et al., 2009. Cancer associated fibroblasts promote tumor growth and metastasis by modulating the tumor immune microenvironment in a 
4T1 murine breast cancer model. PLoS ONE, 4(11):e7965. [doi:10.1371/journal.pone.0007965]

Mendel, D.B., Laird, A.D., Xin, X., et al., 2003. In vivo antitumor activity of SU11248, a novel tyrosine kinase inhibitor targeting vascular endothelial growth factor and platelet-derived growth factor receptors: determination of a pharmacokinetic/pharmacodynamic relationship. Clin. Cancer Res., 9(1):327-337.

Micke, P., Ostman, A., 2005. Exploring the tumour environment: cancer-associated fibroblasts as targets in cancer therapy. Expert Opin. Ther. Targets, 9(6):1217-1233. [doi:10.1517/14728222.9.6.1217]

Motzer, R.J., Hutson, T.E., Tomczak, P., et al., 2007. Sunitinib versus interferon alfa in metastatic renal-cell carcinoma. N. Engl. J. Med., 356(2):115-124. [doi:10.1056/ NEJMoa065044]

Mueller, L., Goumas, F.A., Himpel, S., et al., 2007a. Imatinib mesylate inhibits proliferation and modulates cytokine expression of human cancer-associated stromal fibroblasts from colorectal metastases. Cancer Lett., 250(2): 329-338. [doi:10.1016/j.canlet.2006.10.024]

Mueller, L., Goumas, F.A., Affeldt, M., et al., 2007b. Stromal fibroblasts in colorectal liver metastases originate from resident fibroblasts and generate an inflammatory microenvironment. Am. J. Pathol., 171(5):1608-1618. [doi:10.2353/ajpath.2007.060661]
Orimo, A., Gupta, P.B., Sgroi, D.C., et al., 2005. Stromal fibroblasts present in invasive human breast carcinomas promote tumor growth and angiogenesis through elevated SDF-1/CXCL12 secretion. Cell, 121(3):335-348. [doi:10. 1016/j.cell.2005.02.034]

Raymond, E., Dahan, L., Raoul, J.L., et al., 2011. Sunitinib malate for the treatment of pancreatic neuroendocrine tumors. N. Engl. J. Med., 364(6):501-513. [doi:10.1056/ NEJMoa1003825]

Siegel, R., Ma, J., Zou, Z., et al., 2014. Cancer statistics, 2014. CA Cancer J. Clin., 64(1):9-29. [doi:10.3322/caac.21208]

Tomasek, J.J., Gabbiani, G., Hinz, B., et al., 2002. Myofibroblasts and mechano-regulation of connective tissue remodelling. Nat. Rev. Mol. Cell Biol., 3(5):349-363. [doi:10.1038/nrm809]

Tyan, S.W., Kuo, W.H., Huang, C.K., et al., 2011. Breast cancer cells induce cancer-associated fibroblasts to secrete hepatocyte growth factor to enhance breast tumorigenesis. PLOS ONE, 6(1):e15313. [doi:10.1371/journal. pone.0015313]

Zhang, Y., Tang, H., Cai, J., et al., 2011. Ovarian cancerassociated fibroblasts contribute to epithelial ovarian carcinoma metastasis by promoting angiogenesis, lymphangiogenesis and tumor cell invasion. Cancer Lett., 303(1):47-55. [doi:10.1016/j.canlet.2011.01.011]

\section{中文緊要:}

\section{本文题目：舒尼替尼对结肠成纤维细胞体内外抑制机制研究}

Sunitinib mesylate inhibits proliferation of human colonic stromal fibroblasts in vitro and in vivo

研究目的：探索小分子酪氨酸激酶抑制剂舒尼替尼（sunitinib）对结肠肿瘤微环境中的肿瘤相关成纤维细 胞的作用及其机制。

创新要点：舒尼替尼通过抑制肿瘤间质成纤维细胞的生长，间接发挥抗肿瘤效应，为结肠癌综合治疗的 提供新途径。

研究方法: 通过细胞周期分析和细胞增殖测定进行舒尼替尼体外抑制肿瘤细胞的研究。采用 Western-blot 检测磷酸化血小板衍生生长因子 $\beta$ 受体（PDGFR- $\beta$ ）、蛋白激酶 B (Akt) 及细胞外信号调节 蛋白激酶 (ERK) 的蛋白水平。通过注射肠腺癌细胞株 SW620 和结肠成纤维细胞构建的裸鼠 移植瘤模型来研究舒尼替尼的体内抑瘤效果。

重要结论：舒尼替尼可有效抑制结肠癌来源的原代结肠成纤维细胞生长，该抑制作用主要通过抑制血小 板衍生生长因子（PDGF）信号通路得以实现。

关键词组: 结肠癌; 肿瘤相关成纤维细胞; 舒尼替尼; 血小板衍生生长因子（PDGF）; 血小板衍生生长 因子受体 (PDGFR) 